\title{
ASO Author Reflections: Understanding Subsolid Lung Adenocarcinoma with Cystic Airspaces (LACA)
}

\author{
Woohyun Jung, MD ${ }^{1}$, and Sukki Cho, $\mathrm{MD}^{1,2}$ \\ ${ }^{1}$ Department of Thoracic and Cardiovascular Surgery, Seoul National University Bundang Hospital, Seongnam-si, \\ Gyeonggi-do, Republic of Korea; ${ }^{2}$ Department of Thoracic and Cardiovascular Surgery, Seoul National University College \\ of Medicine, Seoul, Republic of Korea
}

\section{PAST}

Cystic airspaces (CAs) can be associated with lung adenocarcinoma in various ways. ${ }^{1,2}$ The presence of CAs in lung adenocarcinoma was reported to be a poor prognostic factor. ${ }^{3}$ However, it remains unclear whether the CAs are congenital or acquired, whether the presence of CAs precedes the development of lung adenocarcinoma, how they progress, and why they show a poor prognosis.

\section{PRESENT}

According to the recent study by Jung et al., the subsolid lung adenocarcinomas associated with CAs (LACA) showed common radiologic growth pattern from phase I, the appearance of CAs in the middle of a nonsolid nodule, phase II, the enlargement of CAs without change of the thickness of the GGO wall surrounding the CAs, phase III, appearance of solid wall on the boundary of CAs, to phase IV, the solid wall thickening. The higher CEA level, SUV max, pathologic stage, and the grade of the predominant subtype, and more incidence of poor prognostic pathologic findings, such as visceral pleural, vascular and lymphatic invasion, aerogenous spread, and necrosis were identified as LACA progressed from phase I to IV.

(C) Society of Surgical Oncology 2020

First Received: 14 April 2020;

Published Online: 30 April 2020

S. Cho, MD

e-mail: skcho@snubh.org

\section{FUTURE}

Prominent neoangiogenesis, inner cystic wall fibrosis, which is distinct from the stromal reaction and preceded the appearance of the solid component were interesting pathologic findings of LACA, and cancer-associated fibroblasts are an emerging target of anticancer immunotherapy. ${ }^{4}$ Understanding of LACA definitely helps treating LACA itself and would serve as bridgeheads for researching cancer-associated fibroblasts and its treatment.

DISCLOSURES Each author has no conflicts of interest.

\section{REFERENCES}

1. Sheard S, Moser J, Sayer C, et al. Lung cancers associated with cystic airspaces: underrecognized features of early disease. Radiographics. 2018;38:704-17.

2. Mascalchi M, Attinà $\mathrm{D}$, Bertelli $\mathrm{E}$, et al. Lung cancer associated with cystic airspaces. J Comp Assist Tomogr. 2015;39:102-8.

3. Kimura H, Saji H, Miyazawa T, et al. Worse survival after curative resection in patients with pathological stage I non-small cell lung cancer adjoining pulmonary cavity formation. J Thorac Dis. 2017;9:3038-44.

4. Liu T, Han C, Wang S, et al. Cancer-associated fibroblasts: an emerging target of anti-cancer immunotherapy. J Hematol Oncol. 2019;12:86.

Publisher's Note Springer Nature remains neutral with regard to jurisdictional claims in published maps and institutional affiliations. 